\title{
Oral submucous fibrosis in a 11-year-old Bangladeshi girl living in the United Kingdom
}

\author{
B. Shah, ' M. A. O. Lewis, ${ }^{2}$ and R. Bedi, ${ }^{3}$
}

\begin{abstract}
A case of oral submucous fibrosis occurring in a I I-year-old
Bangladeshi girl is presented. This paper reviews the aetiology, clinical presentation and treatment modalities of oral submucous fibrosis. This case highlights the link between oral submucous fibrosis and the regular use of areca-nut (paan) and the newer transcultural oral tobacco products. This association has been reported among children resident in the Indian sub-continent but is unrecorded for United Kingdom residents. The case report underlines the danger that children face with products which are clearly targeted at them by the tobacco industry.
\end{abstract}

$\mathrm{O}_{\mathrm{b}}^{\mathrm{r}}$ al mucosal lesions associated with betel quid, areca nut and tobacco chewing habits, were reviewed at a consensus workshop in Kuala Lumpur, Malaysia in 1994. ${ }^{1}$ Criteria and guidelines were proposed to define, describe and identify lesions such as submucous fibrosis (OSMF) which had been described three decades earlier by Pindborg and Sirsat ${ }^{2}$ as: 'a chronic insidious disease affecting any part of the oral cavity and may extend to the pharynx and the oesophagus, and may be preceded or associated with vesicle formation. It is always associated with juxta-epithelial inflammation and followed by fibro-elastic change of the lamina propria with epithelial atrophy leading to stiffness of the oral mucosa and causing trismus.'

OSMF is seen most frequently in commu-

${ }^{1}$ Specialist Registrar in Orthodontics, Eastman Dental Institute, University College London, 256 Gray's Inn Road, London WCIX 8LD ${ }^{2}$ Professor of Oral Medicine, University of Wales College of Medicine, Heath Park, CardiffCF14 $4 X N^{3 *}$ Professor and Head, National Centre for Transcultural Oral Health, Eastman Dental Institute. University College London, 256 Gray's Inn Road, London WCIX 8LD

${ }^{*}$ Correspondence to: Professor Raman Bedi, National Centre for Transcultural Oral Health, WHO

Collaborating Centre for Disability, Culture and Oral Health, Eastman Dental Institute. University College London, 256 Gray's Inn Road, London WCIX 8LD email:R.Bedi@eastman.ucl.ac.uk

REFEREED PAPER

Received 02.11.00; Accepted 22.03.01

(C) British Dental Journal 2001; 191: 130-132 nities resident in the Indian sub-continent and has a reported incidence of between $0.2-1.2 \%$ of the urban population attending dental clinics. ${ }^{3}$ The condition predominantly affects women with a female: male ratio of $3: 1$ and characteristically first presents in adulthood between the ages of 45-54 years. ${ }^{3}$ Cases of OSMF have also been reported in individuals of 'South Asian' origin living outside the sub-continent but it is extremely rare in White populations. ${ }^{4}$ The present report describes an unusual case of OSMF presenting in a young Bangladeshi child who is resident in the United Kingdom.

\section{Case report}

An 11-year-old Bangladeshi girl was referred to the Oral Medicine Clinic, University Dental Hospital, Cardiff, by her general dental practitioner with a complaint of

In brief

- Oral submucous fibrosis in an I I-year-old Bangladeshi girl, resident in the UK, is reported

- The case highlights the dangers of eating sweetened forms of areca-nut

- Health professionals need to be aware of both the dangers of areca-nut consumption and the newer transcultural tobacco products recurrent oral ulceration. In addition, the patient suffered discomfort and a burning sensation affecting the oral mucosa, particularly when eating spicy foods (Fig. 1). The patient was first seen in December 1994 and her most recent review was September 2000.

Medical history indicated previous deficiency of both iron and vitamin B12. Her iron and vitamin B12 deficiencies were fully investigated by a consultant paediatric gastroenterologist in 1994. There was no abnormality on barium meal and follow-through. On-going review in gastroenterology has been maintained without further findings, but the patient was receiving regular iron supplements and vitamin B12 injections. Previous special investigations for the possible presence of Crohn's disease were negative.

The patient had been born in the United Kingdom and the immediate family had emigrated to the United Kingdom in 1969. The patient has visited Bangladesh on a regular basis and the last visit was in 1998. She was the second eldest of four children. A telephone interview with the father confirmed that there was a long history of tobacco chewing among both parents: the mother is a non-smoker but consumes chewing tobacco 2-3 times per day, the father both smokes and chews tobacco. The patient only occasionally chews betel-quid but regularly chews supari (a sweetened form of areca-nut). This has occurred since the age of 10 years. The patient is aware of the new tobacco products (Gutkha) but did not use these on a regular basis. The parents and all the children were unaware of the harms of supari or chewing tobacco products, believing them to be a natural product which aid digestion.

Intra-oral examination revealed a number of ulcers that were characteristic of recurrent minor aphthous stomatitis. Oral hygiene was poor and the dentition required some restoration. No other soft tissue or mucosal abnormalities were detected at the first examination (December, 1994).

Management involved reassurance and advice on cessation of areca-nut chewing, and chlorhexidine mouth rinse. It was only 
possible to give these instructions directly to the patient herself since her parents, who accompanied her, could not speak English. These instructions and further health education advice, were, via a telephone interview, undertaken by a bilingual translator. A follow-up review at the Oral Medicine Clinic was arranged and the patient referred to the Paediatric Dentistry Unit, University Dental Hospital, Cardiff for routine dental care. Unfortunately, the patient failed to attend several subsequent appointments.

However, the patient did eventually attend 6 months later with a continued history of recurrent aphthous stomatitis. At the age of 13 years, examination revealed that the buccal mucosa was pale and fibrous bands could now be palpated within the buccal mucosa. A clinical diagnosis of OSMF was made and the patient was once again strongly advised to stop the consumption of areca-nut. Prednisolone as $5 \mathrm{mg}$ dispersible tablets was prescribed, to be used as a mouthwash three times daily when ulcers occurred. The oral discomfort had led to poor oral hygiene and there was evidence of pericoronitis associated with a lower second molar. The patient was prescribed metronidazole tablets $200 \mathrm{mg}$ three times daily for 5 days and instructed to use chlorhexidine $0.2 \%$ as a mouthwash twice daily. Regular oral hygiene instruction and scale and polish were arranged.

The patient subsequently failed to attend the Oral Medicine Clinic for almost 2 years before eventually presenting again caused by a difficulty in opening her mouth fully. Medical history at this stage revealed that the iron and vitamin B12 supplements had been stopped. The management of the deficiencies in iron and vitamin B12 had been undertaken by the consultant paediatric gastroenterologist, investigation for thyroid disease by the consultant in paediatrics. To date (September 2000) these investigations had not revealed any abnormality in thyroid function or antibody evidence of autoimmune thyroid disease. On examination, mouth opening was restricted to $18 \mathrm{~mm}$ as measured from incisal edges. Both buccal mucosa and labial mucosa appeared pale, with an obvious increase in evidence of
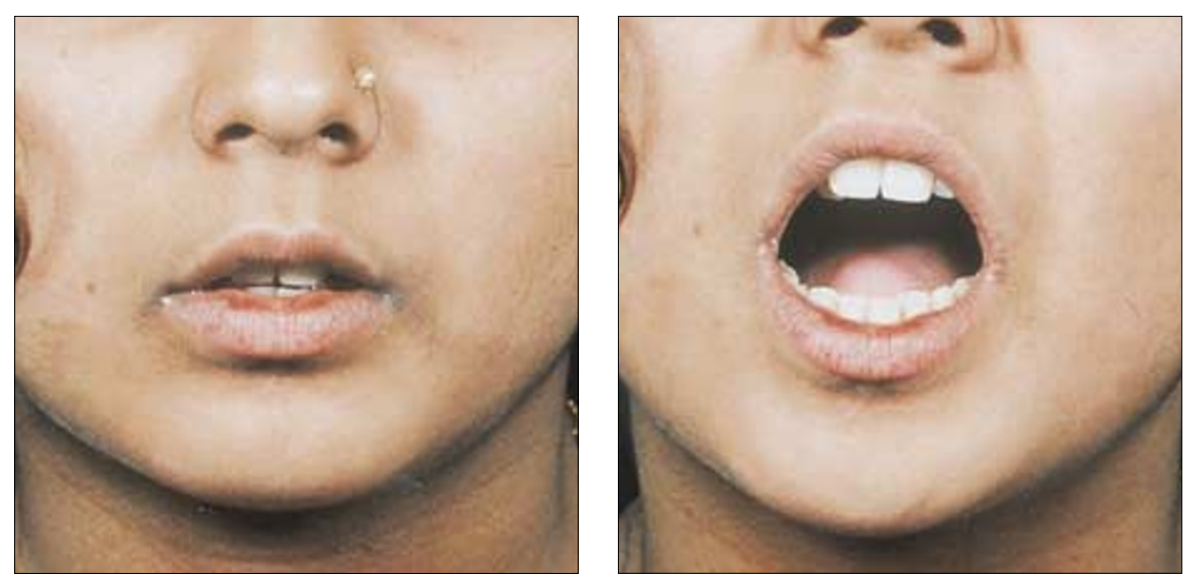

Fig. I a and b I I-year-old Bangladeshi girl showing limited opening associated with the development of oral submucous fibrosis

fibrosis and banding within the cheeks compared with the previous examination.

Unfortunately, this young girl continues to maintain the habit, and in this respect it has to be accepted she is at high risk of developing intra-oral squamous carcinoma in the future.

\section{Discussion}

The aetiology of OSMF remains uncertain and at the present time there is evidence to suggest a combination of factors are likely to be involved. The main proposed factors include the following:

\section{Irritants}

\section{Capsaicin:}

Sirsat and Khanolkar investigated the effect of capsaicin, ${ }^{5}$ a component of chilli peppers, on the palates of Wistar rats and noted a limited connective tissue response, although this was increased when the animals were vitamin B12 deficient.

\section{Acroline, arecaidine:}

Areca-nut is traditionally chewed throughout India as 'paan supari'. The mixture is held adjacent to the buccal mucosa and slowly chewed over a long period of time. Tissue culture studies using human fibroblasts by Harvey et al., ${ }^{6}$ suggests that areca-nut alkaloids, particularly acroline and arecaidine, were involved in causing OSMF. Furthermore it was demonstrated that extracts of arecanut stimulated collagen synthesis by $170 \%$ over the control studies. Many other experimental studies have also shown a strong correlation between OSMF and areca-nut chewing.

\section{Nutritional factors}

Iron and vitamin B12 deficiency has been implicated particularly in conjunction with other factors. In 1919 Paterson and Brown-Kelly independently described the condition of chronic dysphagia and mucosal atrophy in women who had chronic anaemia - this was later termed sidero-penic anaemia or BrownKelly-Paterson Syndrome and has the potential for cancerous change in the oropharynx. $^{7}$

\section{Genetic disposition}

An increased frequency of HLA 10, DR3 and DR7 has been noted in patients with OSMF. $^{7}$

\section{Hormonal factors}

There would appear to be a predisposition in females with a ratio of women to men of $3: 1 .^{7-8}$

\section{Auto-antibodies}

Studies have shown raised levels of a number of immunoglobulins including IgA, IgE 
and IgD. Auto-antibodies to gastric, parietal, and thyroid microsomal and antinuclear antibodies have also been found in $65 \%$ of patients with OSMF. ${ }^{8}$

Diagnosis of OSMF is usually based on the clinical signs and symptoms, which include; oral ulceration, burning sensation (particularly with spicy foods), paleness of the oral mucosa, and occasional leukoplakia. The most characteristic feature is the marked vertical fibrous ridge formation within the cheeks, and board like stiffness of the buccal mucosa. The fibrosis in the soft tissue leads to trismus, difficulty in eating, and even dysphagia. Biopsy of the tissue is rarely performed due to the observation that such investigation results in further fibrous scar formation and worsening of the symptoms. At the present time there is no cure for OSMF and management consists of elimination of the ingestion of implicated irritants. Successful prevention in the early stages of the condition has been shown to produce improvement in symptoms. ${ }^{9}$

In the past 30 years a substantial 'South Asian' population has settled in Britain and therefore it is likely that there will be an increased number of patients with OSMF in the future especially in those individuals who maintain cultural habits that are recognised to predispose to the condition. The close association between areca-nut chewing and the development of OSMF with a risk of oral cancer has been demonstrated in a number of studies. ${ }^{10-11}$ A case control study showed that the condition only occurred among those who chewed areca-nut in one form or another. ${ }^{12}$ However, this observation is still not fully conclusive and other factors such as genetic and nutritional influences also have a role to play. As there is no cure for the condition education and prevention seem the only way to reduce the risk of OSMF in the South Asian community.
The association of the condition with the development of oral cancer highlights the importance of education to limit OSMF. The possible precancerous nature of OSMF was first described by Paymaster, who observed the occurrence of squamous cell carcinoma in one third of his patients with OSMF. ${ }^{10}$ Subsequent studies have reported that the incidence of carcinoma varies in OSMF from 2-30\%. ${ }^{13}$

More recently the chewing of gutkha; a sweetened mixture of tobacco and betel-nut, has increased in this country and its use is thought to be commercially aimed at children. ${ }^{14}$ Since gutkha has only been widely available in the UK for the past 3 years, there is uncertainty over the long-term potential adverse effects of this product. ${ }^{14}$

The present report describes the presentation of OSMF in childhood. However, dental practitioners should be reminded of the condition since it may well be seen more frequently in the future and an active preventative approach is required to hopefully limit the potential for the development of oral malignancy.

\section{Conclusion}

OSMF in the young girl described in the present case was difficult to manage in part caused by her age and also caused by a communication problem with her parents due to a language barrier. In addition she was found to be a poor attender and this made monitoring and managing the earliest stages of the condition difficult. It seems inevitable that her OSMF is likely to worsen since she would not appear to be able to stop the ingestion of areca-nut. If the condition does worsen, she may in the long term need surgical intervention with grafting, ${ }^{15}$ and there is always the possibility of malignant change, and therefore close monitoring of her oral mucosa is essential. ${ }^{15}$
1 Zain R B, Ikeda N, Gupta P C, Warnakulasuriya S, van Wyk C W, Shrestha P, Axell T. Oral mucosal lesions associated with betel quid, areca nut and tobacco chewing habits: consensus from a workshop held in Kuala Lumpur, Malaysia. November 25-27, 1996. J Oral Path Med 1999: 28: 1-4.

2 Pindborg J J, Sirsat S M. Oral Submucous Fibrosis. Oral Surg Oral Med Oral Pathol 1966; 22: 764-779.

3 Jayanthi V, Probert C S J, Sher K S, Mayberry J F. Oral Submucous Fibrosis: a preventable disease. GUT 1992; 33: 4-6.

4 Murti P R, Bhonsle R B, Gupta P C, Daftary D K, Pindborg J J, Mehta F S. Aetiology of Ora Submucous Fibrosis with special reference to the role of areca nut chewing. J Oral Pathol Med 1995; 24: 145-152.

5 Sirsat S M, Khanolkar V R. Oral Submucous Fibrosis of the palate in diet preconditioned Wistar rats. Arch Path 1960; 70: 171-179.

6 Harvey W, Scutt A, Meghji S, Canniff J P. Stimulation of human buccal mucosa fibroblasts in vitro by betel-nut alkaloids. Arch Oral Biol 1986; 3: 45-49.

7 Pillai R, Balaram P, Reddiar K.S. Pathogenesis of Oral Submucous Fibrosis. Cancer 1992; 69: 2011-2017.

8 Canniff J P, Harvey W, Harris M. Oral Submucous Fibrosis: its pathogenesis and management. Br Dent J 1986; 160: 429-434.

9 Hayes P A. Oral Submucous Fibrosis in a 4 year old girl. Oral Surg Oral Med Oral Pathol 1985; 59: 475-478.

10 Paymaster J.C. Cancer of the buccal mucosa: a clinical study of 650 cases in Indian patients. Cancer 1956; 9: 431-435.

11 Pindborg J J. Is Submucous Fibrosis a precancerous condition in the oral cavity? Int Dent J 1972; 22: 474-480.

12 Sinor P N, Gupta P C, Murti P R et al. A case control study of Oral Submucous Fibrosis with special reference to the aetiological role of areca nut. J Oral Pathol Med 1990; 19: 94-98.

13 McGurk M. and Craig G T. Oral Submucous Fibrosis: 2 cases of malignant transformation in Asian Immigrants. Br J Oral Max-fac Surg 1984; 22: 56-64.

14 Bedi.R. What is Gutkha? BDA News April 1999; 12: 20-21.

15 Rajendran R. Oral Submucous Fibrosis: aetiology, pathology and future research. Bull WHO 1994; 72: 985-996. 